\title{
Article
}

\section{Assessment of Periodontium Temperature Changes under Orthodontic Force by Using Objective and Automatic Classifier}

\author{
Monika Machoy ${ }^{1, *(D)}$, Liliana Szyszka-Sommerfeld ${ }^{1}$, Robert Koprowski ${ }^{2}$, Anna Wawrzyk ${ }^{3}$, \\ Krzysztof Woźniak ${ }^{1}$ and Sławomir Wilczyński ${ }^{4}$
}

1 Division of Orthodontics, Pomeranian Medical University in Szczecin, Powstańców Wielkopolskich Street 72 , 70-111 Szczecin, Poland; liliana.szyszka@gmail.com (L.S.-S.); krzysztof.wozniak@pum.edu.pl (K.W.)

2 Department of Biomedical Computer Systems, Faculty of Computer Science and Materials Science, Institute of Computer Science, University of Silesia, 40-007 Katowice, Poland; robert.koprowski@us.edu.pl

3 Sanitary-Epidemiological Station, Prądnicka 76, 31-202 Kraków, Poland; anna.wawrzyk@gazeta.pl

4 Department of Basic Biomedical Science, Faculty of Pharmaceutical Sciences in Sosnowiec,

Medical University of Silesia, Kasztanowa Street 3, 41-200 Sosnowiec, Poland; swilczynski@sum.edu.pl

* Correspondence: m.machoy@gmail.com

check for

updates

Citation: Machoy, M.;

Szyszka-Sommerfeld, L.; Koprowski,

R.; Wawrzyk, A.; Woźniak, K.;

Wilczyński, S. Assessment of

Periodontium Temperature Changes under Orthodontic Force by Using Objective and Automatic Classifier. Appl. Sci. 2021, 11, 2634. https:// doi.org/10.3390/app11062634

Academic Editor: Yahan $\mathrm{Hu}$

Received: 25 January 2021

Accepted: 11 March 2021

Published: 16 March 2021

Publisher's Note: MDPI stays neutral with regard to jurisdictional claims in published maps and institutional affiliations.

Copyright: (c) 2021 by the authors. Licensee MDPI, Basel, Switzerland. This article is an open access article distributed under the terms and conditions of the Creative Commons Attribution (CC BY) license (https:/ / creativecommons.org/licenses/by/ $4.0 /)$.

\begin{abstract}
Background: Orthodontic elastics are used in orthodontic treatment with fixed braces The effect of class I elastics on changing gingiva temperature as a periodontal component was investigated. Methods: To evaluate the temperature changes, objective and automatic classifiers were used to determine the sensitivity and specificity of the measurement. Results: Before putting on the elastics, the average gingival temperature of all 120 patients was $34.72 \pm 0.7548^{\circ} \mathrm{C}$. Putting on the elastics for $10 \mathrm{~min}$ slightly increased the temperature to $34.81 \pm 0.5938^{\circ} \mathrm{C}$; however, this is not a statistically significant change $(p>0.05)$. Conclusion: It is concluded that putting an elastic band on does not significantly affect the gingiva temperature change. The use of class I elastics in orthodontic treatment in patients with healthy periodontium does not significantly change the periodontal temperature, which indirectly proves the production of safe orthodontic forces that can be used in the clinic. The use of artificial intelligence in the assessment of the temperature of the gingiva makes it possible to exclude factors that may disturb the objective thermographic analysis.
\end{abstract}

Keywords: thermal imaging; image analysis; periodontium; orthodontic treatment; orthodontic braces

\section{Introduction}

Periodontium as a component of the masticatory organ surrounds and immobilizes the tooth. It consists of root cementum, alveolar bone with periosteum, periodontium, and gingiva [1]. During orthodontic treatment, permanent forces applied to the tooth are transferred to the periodontium. With a significant load on it, immediate movement within the periodontal space is prevented by tissue fluid. During the first second of pressure on the tooth, a small amount of tissue fluid is squeezed out of the periodontal space. If the pressure on the tooth persists, the fluid is squeezed out quickly, and the tooth moves inside the periodontal gap by pressing the periodontal ligament to the adjacent bone. Normally, 3-5 s after applying a great force, pain is felt, which indicates that the tissue fluid has been squeezed out and the crushing pressure acts on the periodontal ligament itself [2].

The periodontal ligament, due to the special arrangement of collagen fibers, is well adapted to resist forces that act briefly, such as chewing forces, which reach 700-1000 N within molars [3-5]. The maximal bite force clearly depends on many factors such as gender, number of interdental contacts in the occlusion, and muscles size [6]. However, these are intermittent forces-during chewing, tooth contact lasts a maximum of 1 s. Constant forces cause such a strong displacement of the tooth within the periodontal gap that the tissue fluid is squeezed out of the periodontal space. Orthodontic force, acting for a long time, 
despite low intensity, causes periodontal remodeling, resulting in resorption and aposition of bone tissue belonging to the periodontium. The response to orthodontic force applied to the teeth is a function of the magnitude of the force [2,7-11].

After applying orthodontic forces, the tooth moves in the periodontal ligament under the force of pressure from one side of the tooth root and pulling on the other. These forces change the blood flow within the blood vessels - the pressure of the tooth on the periodontal gap decreases the blood flow and pulling increases by changing the cross-section of the blood vessels. Changes in blood flow in the oppressed area cause a decrease in oxygen levels, and in the tense part of the ligament, it increases. The appropriate proportions of metabolites change within a few minutes. After 1-2 s, the fluid is already partly squeezed out, and the tooth moves within the periodontal space. The blood vessels within the periodontium are partially narrowed on the compressed side $3-5 \mathrm{~s}$ from the application of force and widened on the stretched side; the fibers and periodontium cells are mechanically twisted, which is a sign of toothache. After a few minutes, the blood flow changes, oxygen pressure begins to change, and prostaglandins and cytokines are released [12]. The action of force affects the systemic remodeling of the appendix and periodontiums up to 14 days from the application of force due to the activation of cells responsible for resorption and bone superstructure [13]. From the point of view of physiology, periodic metabolic activity during tooth movement is associated with limited inflammation of the above tissues. The temperature of a healthy gingiva measured subgingivally in the space between the marginal gingiva and the connecting epithelium is on average $36.1^{\circ} \mathrm{C}$ [14]. Considering this fact, the following studies hypothesized that a constant orthodontic force applied to the teeth, by changing the blood flow and affecting the release of proinflammatory cytokines, affects the change in periodontal temperature and thus also the gingiva. Theoretically, within the part of the pulled periodontal ligament, the temperature should change differently than in the part where collagen fibers and vessels are compressed.

The article below aims to assess the change in gingiva temperature as a component of periodontium under the influence of short-term but constant orthodontic force and to develop a novel prediction model for gingiva thermograms acquisition using an objective and automatic classifier.

The use of thermovision measurements in the quantitative analysis of the temperature of mucous membranes in response to a mechanical factor (elastomeric ligature) faces a number of difficulties. These include inconvenient access to the examined spot, the impact of other factors on the gingiva temperature outside the stimulus-elastomeric band-among others, possible inflammation of the gingiva, difficulties in precisely determining characteristic points to compare the temperature before and after the application of the eraser, and relatively small differences in temperature change are expected from the response to the given band. Therefore, to minimize the matching error and the impact of other situations mentioned above that may affect the final effect of thermovision measurement, classifiers were used to determine the sensitivity and specificity of the measurement.

\section{Materials and Methods}

The patients were obtained under a protocol that had been analyzed and approved by the Ethical Committee of the Pomeranian Medical University, Szczecin, Poland. The number of the resolution: KB-0012/85/2020. Patients were examined during a standard orthodontic visit, which therefore lasted longer and which they were informed about before coming to the facility.

\subsection{Patient's Inclusion Criteria}

A total of 120 patients treated orthodontically with permanent braces were qualified for the study.

Inclusion criteria involved the following:

1. All teeth in the arch.

2. No inflammation of the gingiva and periodontium evaluated by 
- $\quad$ Plaque Index (PII) according to Silness and Löe. Index equal to 0-1;

- API (Aproximal Plaque Index) according to Lange. Index equal to $0-25 \%$.

- $\quad$ GI (Gingival Index) according to Löe and Silness. GI equal to 0;

- SBI (Sulcus Bleeding Index) according to Muhemann and Son. SBI Index equal to 0;

- Absence of tartar (supragingival and subgingival), periodontal condition (bleeding, soreness, swelling, redness, mobility and migration of teeth, gingival recessions, unpleasant odor).

3. No general medical related to systemic diseases history: infectious (hepatitis, HIV, tuberculosis), allergic (allergic asthma, drug allergies), metabolic (diabetes) and cardiovascular diseases, patients of childbearing age were also asked about pregnancy.

4. Orthodontic treatment in the contracting or equalization phase, orthodontic brackets 0.018 'MBT, arches $0.016 \times 0.022$ SS or $0.017 \times 0.022$ SS.

5. Age $18-35$ years.

The following studies did not take into account gender.

\subsection{Indexes}

Plaque Index (PII Index)

The PII index, determining the thickness of dental plaque located in the region of the tooth cervix on four surfaces, was calculated at teeth 16, 11, 24, 36, 31, and 44 .

Plaque thickness was determined on a four-point PII scale:

0 -no plaque;

1-a thin layer of plaque adjacent to the gingival margin and cervix of tooth; detected by dental probe but invisible to the naked eye;

2-moderate plaque buildup at the edge of the gingiva and/or on the surface; tooth and the gingival pocket visible to the naked eye;

3 -abundant accumulation of plaque in the gingival pocket and/or on the edge gingiva and tooth surfaces.

The modified API index determines the percentage of plaque area. An examination to detect plaque is performed with a probe inserted into the interdental space.

The assessment was carried out on four areas:

- Palatal surface of teeth in the upper right quadrant;

- $\quad$ Labial and malar surface of teeth in the upper left quadrant;

- $\quad$ Lingual surface of teeth in the lower left quadrant;

- Labial or cheek surface of teeth in the lower right quadrant.

The API was calculated according to the following formula:

$$
A P I=\frac{\text { sum of interdental spaces with dental plaque }}{\text { sum of all interdental spaces }} \cdot 100 \%
$$

The hygiene in the examined patients was assessed depending on the percentage of area on which dental plaque was detected. Based on the calculated values of the API index, individual patients were classified into one of four categories defining the state of oral hygiene, which was originally proposed by the creator of the Lange index:

1 API $<25 \%$ optimal oral hygiene, providing protection against tooth decay and periodontal disease.

2 API $=25-35 \%$, very good oral hygiene; the values are sufficient for systematic treatment of caries and periodontal disease, as they ensure the prevention of these diseases.

3 API $=35-70 \%$ moderate oral hygiene.

4 API $=70-100 \%$ inadequate oral hygiene.

The GI index

The GI was assessed on teeth $16,11,24,36,31$, and 44 . The assessment was made in relation to the four-grade scale of the gingiva condition proposed by the authors: 0 -healthy gingiva, pale pink; 1 -mild inflammation, slight changes in gingiva color, and mild tissue 
structure changes, no bleeding; 2-moderate inflammation, redness, swelling, gloss, and hypertrophy of the gingiva, bleeding on pressure or sampling; 3-severe inflammation, significant redness and swelling of the gingiva, ulceration, tendency to spontaneous bleeding.

The SBI gingival index

The SBI allows to capture the early stages of periodontal disease. Gingival assessment was performed on teeth $16,11,24,36,31$, and 44 , and in the case of missing or missing teeth 24 and/or 44, the gingiva were assessed on teeth 26 and 46. Gingival examination consisted of careful probing of the gingival gap, observation of color, and configuration gingiva, and then qualifying for one of the categories on a six-point scale: 0 - healthy gingiva, no bleeding during probing; 1 -healthy-looking gingiva but bleeding when probing the gingival gap; 2-change in gingiva color, bleeding under the influence of irritants; 3 -light change in shape, change in color, bleeding under irritation; 4-significant change in shape, change in color, and bleeding under the influence of irritants; 5-significant swelling, change in color, bleeding gingiva during probing, and possible ulceration of the gingiva.

Between the canine and the first molar of each quadrant of the jaws, one-jaw 1st class orthodontic elastics with a diameter of $4 \mathrm{~mm}\left(1 / 8^{\prime \prime}\right)$ with a pulling force of $110 \mathrm{~g}$ was stretched. The force was measured for each lift with an orthodontic dynamometer specially designed for measuring the pull force (DO.18,000, Falcon, Poland).

\subsection{Image Taking, Analysis and Processing}

The mean room temperature was $20.8 \pm 1.21{ }^{\circ} \mathrm{C}$, and the mean humidity was $53.67 \pm 5.98 \%$. These values are within the range specified by the manufacturer of the infrared camera for optimum measurement conditions.

The measurement parameters were each time calibrated with respect to the environmental conditions (temperature, humidity, distance from the object).

The use of infrared analysis to evaluate the gum temperature required minimizing the measurement errors resulting from:

- Wrong estimation of the object emissivity;

- Determination of the IR radiation reflected from objects in the environment of the test

- subjects;

- Atmospheric attenuation, dispersion, and mission;

- Changes in the radiation of optical camera components;

- Incorrect methodology for the measurement procedure;

- Convection of air currents;

- Patient's emotional state;

- Effect of the patient's clothes;

- Thermal conductivity from limited and diffused sources of heat;

- Skin vascularization;

- Meals consumed in the past $24 \mathrm{~h}$;

- Crossed radiation;

- Undisclosed diseases of probands.

All these aspects were taken into consideration in the acquisition of thermal images of the volunteers' gingiva.

The acquisition of thermal images was performed using a FLIR T420sc infrared camera (FLIR Systems, Inc., Wilsonville, OR, USA).

The data acquisition and analysis were performed using the FLIR ResearchIR version 3.5 (FLIR Systems, Inc., Wilsonville, OR, USA) and Matlab version 12.0 (The MathWorks, Inc., Natick, MA, USA).

The thermal camera from which images were acquired was Flir T420SC infrared camera providing a sequence of $2 \mathrm{D}$ images with a spatial resolution of $240 \times 320$ pixels and a temperature resolution of $0.1 \mathrm{o} \mathrm{C}$. The wavelength range was from 7.5 to $13 \mathrm{um}$, frame rate $60 \mathrm{~Hz}$ and thermal sensitivity (N.E.T.D) $<0.045^{\circ} \mathrm{C}$ at $30^{\circ} \mathrm{C}$. The software used in the 
camera was Flir Researchir 4.1, the image processing analysis was conducted in Matlab ver. $2019 b$.

The camera used in the research is approved for use in medicine. The camera has a valid calibration certificate. The emissivity of the camera is optimized for measuring the temperature of human tissues. Moreover, the spectral range of the camera, $7.5-13 \mu \mathrm{m}$, is optimal for measuring human thermal parameters.

The sequence of images from the thermal imaging camera was saved in the form ${ }^{*}$ mat at a resolution of $\mathrm{M} \times \mathrm{N} \times \mathrm{I}=240 \times 320 \times 300$ pixels (where $\mathrm{M}$-number of rows, $\mathrm{N}$-number of columns, I-number of frames). Each pixel of the image is recorded in 8-bit resolution with an accuracy of $0.1^{\circ} \mathrm{C}$. Image analysis consists of three main parts:

- Image preprocessing;

- Analysis and processing of the gingiva area;

- Extract features and compare it with a pattern.

\subsection{Initial Image Processing}

In the initial image processing, the input image Lw $(m, n)$, where m-rows, n-columns, is the basis for the analysis. Due to the specifics of camera operation and pixel calibration, thermal imaging images are denatured by median filters. Due to the minimum size of a single cluster of artifacts not exceeding 4 pixels, a median filter with a mask size of hs $=9 \times 9$ pixels was used. The second filtration was carried out in the area of the third coordinate (I-th) related to time (image sequence). The mask size for time domain filtration was based on previous analyzes and experiments, hd $=3 \times 1$ pixels.

\subsection{Analysis and Processing of the Gingiva Area}

The image sequence after the initial Le $(m, n, i)$ filtration is further processed using mathematical morphology, conditional opening and closing methods allowing the separation of the lower and upper gingiva in the Le $(m, n, i)$ image sequence. The threshold value necessary for erosion and conditional dilatation was adopted at the level of $\mathrm{pr}=0.5$. The image after erosion and conditional expansion of joints Ledw $(m, n)$ was further subjected to GLCM analysis to determine Contrast Energy Homogeneity. These features formed the basis for segmentation of gingiva objects. The separated objects and their features (Contrast, Energy, Homogeneity) were compared with the standard. The standard was a uniform temperature distribution with the average value calculated for the first frames of the Lw images $(m, n)$. Based on this isolated ROI, sensitivity (TPR), specificity (SPC), and efficacy (ACC) values were calculated based on results (false positive-FP, false negative-FN, true positive-TP, true negative-TN). Four different classifiers were selected for testing: binary decision trees, naive Bayes classifier, discriminant analysis, and support vector machine (SVM). The results obtained are shown in Table 1. Block diagrams of the image analysis and processing algorithm are depicted in the block diagram in Figure 1.

Table 1. Results obtained for 120 healthy patients and 120 treated patients. The measurements of the diagnostic value of the classifiers used. $\mathrm{TP}$-true positive; $\mathrm{TN}$ - true negative; $\mathrm{FP}$-false positive; FN—false negative; TPR—-true positive rate; SPC—specificity; ACC—calculating accuracy.

\begin{tabular}{cccccccc}
\hline & TP & TN & FP & FN & TPR & SPC & ACC \\
\hline Classifier type/parameter & 94 & 98 & 22 & 26 & 0.78 & 0.81 & 0.8 \\
\hline Decision trees & 102 & 104 & 16 & 18 & 0.85 & 0.86 & 0.85 \\
\hline Naive Bayes classifier & 111 & 117 & 3 & 9 & 0.92 & 0.97 & 0.95 \\
\hline Discriminant Analysis & 84 & 93 & 27 & 36 & 0.7 & 0.77 & 0.73 \\
\hline
\end{tabular}




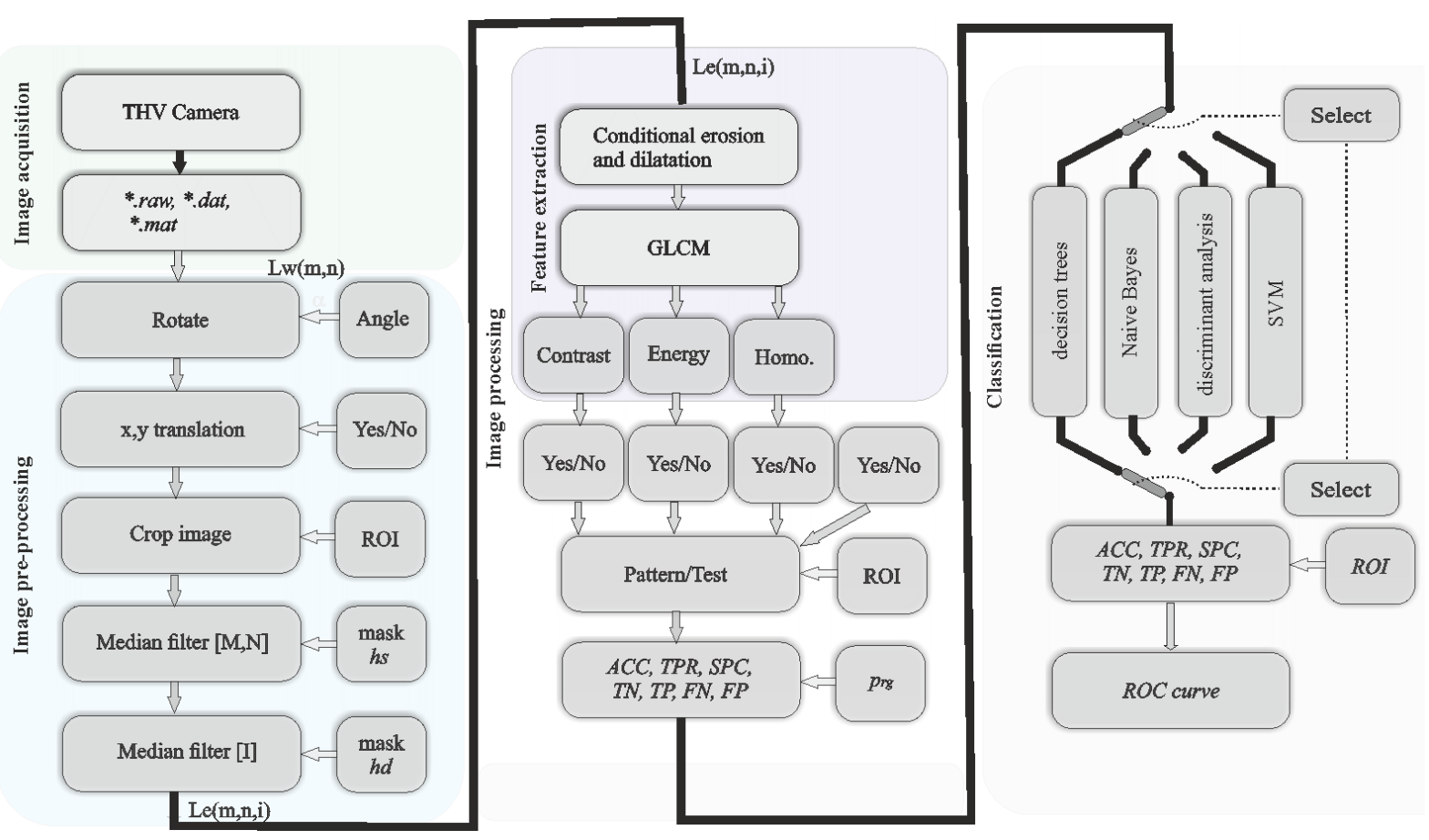

Figure 1. Block diagram of the image analysis and processing algorithm.

\subsection{Data Categorization}

In order to determine the sensitivity and specificity of the classifiers used in assessing the impact of rubber bands on the gingiva temperature, it was necessary to determine which independent variables (predictors) best divide a given set of cases into naturally occurring groups described by a qualitative dependent variable. In order to build a model (classifier) describing a predefined set of data classes, it was also necessary to determine the gingiva temperature in response to a mechanical factor using a method recognized as the gold standard. In this case, the TG56, FLIR pyrometer with a laser pointer was used, as well as physical and subjective examination using qualitative scales carried out by a specialist orthodontist.

In order to compare measures of the diagnostic value of classifiers, such concepts as:

True-Positive (TP): positive prediction, actually observed positive class;

True-Negative (TN): negative prediction, actually observed negative class;

False-Positive (FP): positive prediction, actually observed negative class;

False-Negative (FN): negative prediction, actually observed positive class;

TRP true positive rate: measures the proportion of actual positives that are correctly identified;

$$
T R P=\frac{\sum \text { True positive }}{\Sigma \text { Condition positive }}
$$

Specificity (SPC): measures the proportion of actual negatives that are correctly identified;

$$
S P C=\frac{\sum \text { True negative }}{\Sigma \text { Condition negative }}
$$

Accuracy (ACC): is the proportion of true results, either true positive or true negative, in a population.

$$
A C C=\frac{\Sigma \text { True positive }+\Sigma \text { True negative }}{\Sigma \text { Total population }}
$$




\section{Results}

Samples of the selected images from a sequence of images are presented on Figure 2. The thermal image of the tissue before the treatment is presented on Figure 3. The results of reconstruction of the three areas most important from the point of view of the analysis for $\mathrm{T} 1=29, \mathrm{~T} 2=32$, and $\mathrm{T} 3=34{ }^{\circ} \mathrm{C}$ are presented in Figures 4 and 5 .
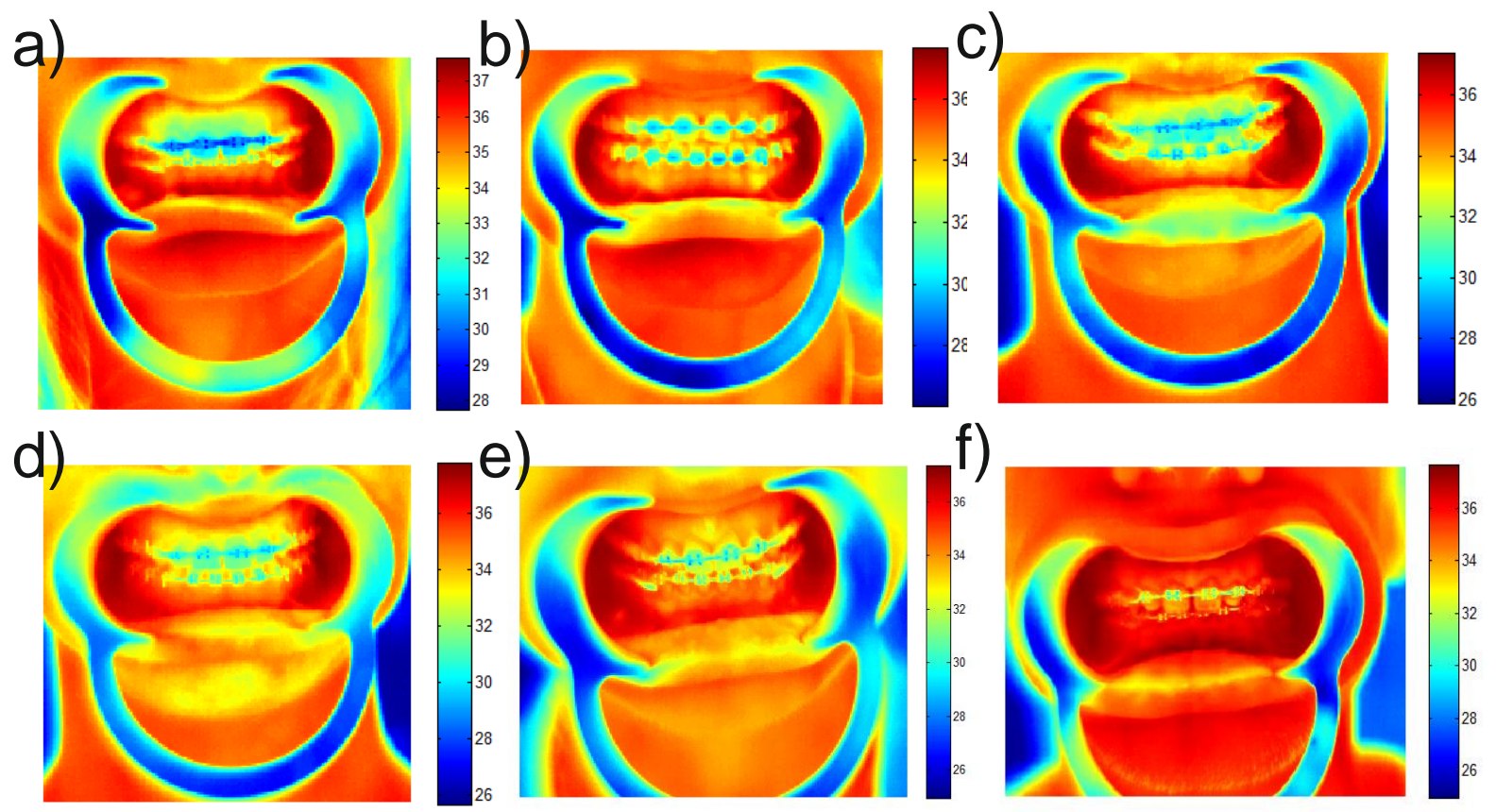

Figure 2. Sample, selected images from a sequence of images, before measurements for (a) patient No. 1; (b) patient No. 2; (c) patient No. 3 ; (d) patient No. 4 ; (e) patient No. 5; (f) patient No. 6.

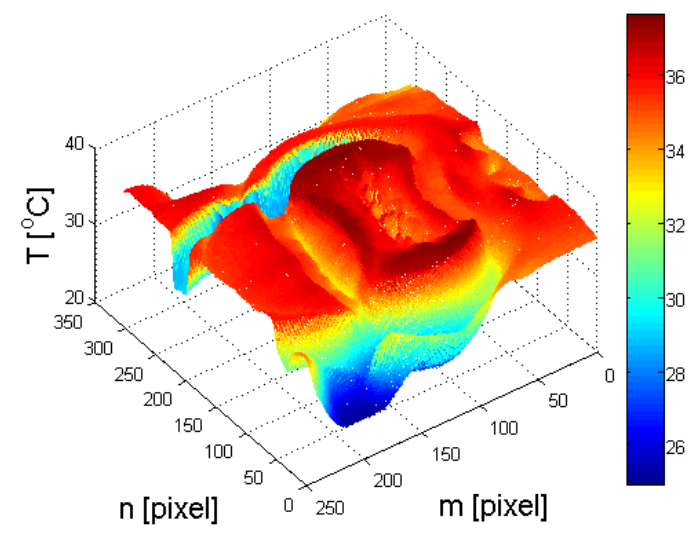

Figure 3. An example of a thermal image made before the test.

The Le $(m, n, i)$ sequence of images prepared in this way is the basis for further processing and extraction of the gingival area.

Table 1 shows the measurements of the diagnostic value of the classifiers used. Discriminant analysis was characterized by the highest sensitivity and the support vector machine by the lowest sensitivity. The same results were obtained for specificity and accuracy

Support vector machine generated the most false-negative results- $15 \%$ of results. In turn, discriminant analysis generated the least false-negative results—only 3.75\%. Discriminant analysis also proved to be very effective in terms of false positive results—only $1.25 \%$ of the results in the whole surveyed population. 


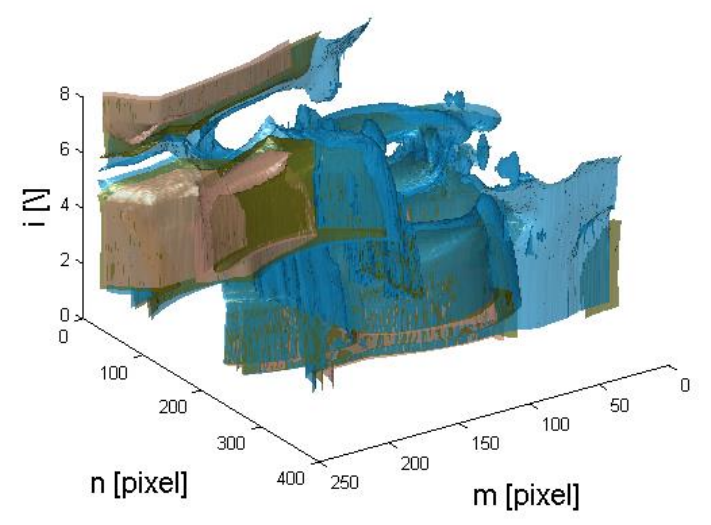

Figure 4. Reconstruction of the three areas most important from the point of view of analysis for $\mathrm{T} 1=29, \mathrm{~T} 2=32$, and $\mathrm{T} 3=34{ }^{\circ} \mathrm{C}$.

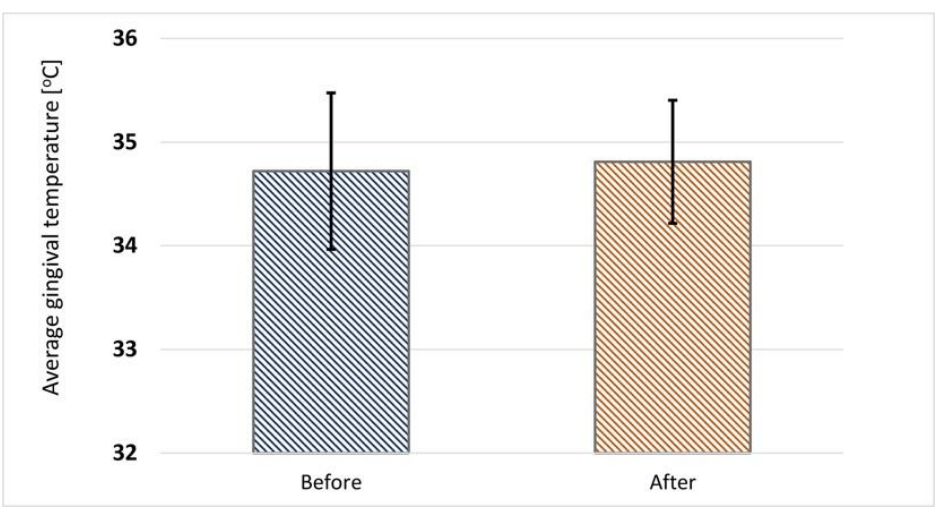

Figure 5. Box plot showing the difference in mean gingival temperature at the beginning of the test and at the end-before putting on the elastics and after $10 \mathrm{~min}$ of its acting.

It should be emphasized that putting an elastic band on does not significantly affect the gingiva temperature change. Before putting on the elastics, the average gingival temperature of all 120 patients was $34.72 \pm 0.7548^{\circ} \mathrm{C}$. Putting on the elastics for $10 \mathrm{~min}$ slightly increased the temperature to $34.81 \pm 0.5938^{\circ} \mathrm{C}$; however, this is not a statistically significant change $(p>0.05)$.

\section{Discussion}

The results of the presented tests show clinical but a statistically insignificant difference in the temperature measurement results. This proves that the use of class I orthodontic elastics, despite their effectiveness in treatment, does not have a negative impact on the health of the periodontium, where an increase in temperature by as little as $0.5^{\circ} \mathrm{C}$ indicates periodontitis [14]. The rise in temperature in the affected sites may reflect complex cellular, molecular, and metabolic events during the inflammatory response. This means that an increase in cellular activity generates heat that heats the blood flowing through this site, thus causing an increase in temperature in the periodontal pocket. The results of the presented tests will be reproducible regardless of the anatomical area of the jaw and mandible-temperature changes will be the same in the anterior and posterior areas, both the upper jaw and the lower jaw, because the study of temperature changes depending on the anatomical area of the appendages and alveolar parts. Gunupati et al. tested temperature differences between anterior and posterior regions and between mandibular and maxillary arches, and the changes were clinically observed, but the differences were not statistically significant [15]. The effect of elastomers on gingival temperature was also investigated by Azan et al. They tested the influence of fluorinated elastic ligatures on the temperature increase of the gingiva as the marginal part of the periodontium. They noticed a statistically significant increase in temperature of $0.3{ }^{\circ} \mathrm{C}$, which, like in our 
research, however, cannot be considered clinically significant. The increase in temperature of the tooth gingiva on which the elastic is based occurred regardless of the type of elastic. The mechanism has not been fully understood yet, but it has been assumed that it is related to the ligature swelling under the influence of saliva, increasing its roughness, and the formation of retention sites for bacteria causing contact inflammation. In our study, inflammation was ruled out during physical examination, and the increase in temperature is associated only with the production of pressure on the periodontium.

Studies on the effect of orthodontic forces [16-18], including the effect of elastics on the periodontium, have been studied for years $[19,20]$.

The proposed method of thermovision measurements supported by methods of image analysis and processing allows for quantitative identification of the gingiva temperature in response to a mechanical factor which is the fitting of an eraser. Due to the fact that gingival temperature measurement can disturb a number of factors including difficult access to the gingiva, the effect of salivary secretion, relatively small expected temperature difference, expected large individual variability in temperature response to the mechanical factor, and difficulties in verifying the ROI (region of interest) - pairing of images before and after putting on orthodontic elastics to determine the temperature difference within the same location on the gingiva-it was proposed to use classifiers that allow the quantitative verification of the possibility of using thermovision in the examination of gingival mucosa temperature.

The proposed method of analyzing thermographic results indicates that there is no statistically significant difference $(p>0.05)$ in the gingiva temperature within the teeth on which the erasers were applied after $10 \mathrm{~min}$. This indicates that the temperature changes that were recorded in publication are not caused by the directly applied mechanical force acting directly on periodontal tissues but by other physiological factors associated with the long-term process of bone tissue remodeling. Therefore, it is not possible to use thermography in the ad hoc test to assess the degree of impact of elastic bands on the teeth, and thus, it is not possible to correlate the elasticity of the orthodontic elastics (Young module) with the temperature obtained within the gingiva. Therefore, thermography is not an effective method that allows the selection and verification of elasticity of elastics and thus the immediate effect of the apparatus on the teeth. It should also be emphasized that measuring periodontal temperature in response to a given mechanical factor causes many difficulties. These include hardly accessible location, small expected difference in temperature change obtained, influence of other factors on the gingiva temperature change besides the applied mechanical force, and difficulties in finding characteristic points on the gingiva to proactively determine identical ROIs on the corresponding image sequences (before and after putting on elastics).

In view of the above, to verify the possibility of using thermovision measurements of the gingiva, machine learning methods have been proposed, which allow determining the diagnostic value measures of the classifiers used. The support vector machine shows the lowest sensitivity, specificity, and accuracy in the proposed test model. In this case, the support vector machine allows predicting whether the measurement conditions, and the results obtained are a reliable source of medical data with a sensitivity of $70 \%$, a specificity of $77 \%$, and a sensitivity of $73 \%$. In view of the above, it should be noted that the thermographic measurement of gingiva, despite the precise determination of teaching vectors and several-step verification of attributes in the teaching group (temperature measured by a pyrometer, physical, and subjective tests performed by an orthodontics specialist using qualitative scales), can be significantly disturbed by numerous factors on the final result of the measurements. The proposed classifiers allow to assess whether the temperature changes within the periodontium were associated with the placement of elastics or other factors and to determine the patient's stabilization relative to the thermal imaging camera, which is significant in the case of in vivo measurements in hard-to-reach anatomical locations. 


\section{Conclusions}

1. The use of class I elastics in orthodontic treatment in patients with healthy periodontium does not significantly change the periodontal temperature, which indirectly proves the production of safe orthodontic forces that can be used in the clinic.

2. The use of artificial intelligence in the assessment of the temperature of the gingiva makes it possible to exclude factors that may disturb the objective thermographic analysis.

Author Contributions: Conceptualization, M.M.; methodology, M.M.; software, S.W.; validation, L.S.-S., K.W., A.W.; formal Analysis, A.W.; investigation, M.M., S.W.; resources, K.W.; data curation, M.M., R.K.; writing original draft preparation, M.M.; writing review and editing, M.M.; visualization, S.W.; supervision, S.W., K.W.; project administration, M.M.; funding acquisition, K.W., S.W. All authors have read and agreed to the published version of the manuscript.

Funding: This study has been financially supported by Medical University of Silesia, grant no. PCN-1-013/K/0/O.

Institutional Review Board Statement: The study was conducted according to the guidelines of the Declaration of Helsinki, and approved by the Institutional Review Board (or Ethics Committee) of Pomeranian Medical University (KB-0012/85/2020).

Informed Consent Statement: Informed consent was obtained from all subjects involved in the study.

Data Availability Statement: The data presented in this study are available on request from the corresponding author.

Conflicts of Interest: The authors have no competing interests to declare.

\section{References}

1. Zabel, M. Histologia: Podręcznik dla Studentów Medycyny i Stomatologii; Elsevier Urban \& Partner: Wrocław, Poland, 2000; ISBN 97883-87944-61-2. (In Polish)

2. Khouw, F.E.; Goldhaber, P. Changes in vasculature of the periodontium associated with tooth movement in the rhesus monkey and dog. Arch. Oral. Biol. 1970, 15, 1125-1132. [CrossRef]

3. Mainland, A.; Hiltz, J.E. Forces exerted on the human mandible by the muscles of occlusion. J. Dent. Res. 1934, 14, 107-124. [CrossRef]

4. Osborn, J.W.; Baragar, F.A. Predicted pattern of human muscle activity during clenching derived from a computer-assisted mode: Symmetric vertical bite forces. J. Biomech. 1985, 18, 599-612. [CrossRef]

5. Koolstra, J.H.; van Eijden, T.; Weijs, W.A.; Naeije, M. Three-dimensional mathematical mode of the human masticatory system predicting maximum possible bite forces. J. Biomech. 1988, 21, 563-576. [CrossRef]

6. Ahlberg, J.P.; Kovero, O.A.; Hurmerinta, K.A.; Zepa, I.; Nissinen, M.J.; Könönen, M.H. Maximal bite force and its association with signs and symptoms of TMD, occlusion, and body mass index in a cohort of young adults. Cranio 2003, 21, 248-252. [CrossRef] [PubMed]

7. Dorow, C.; Schneider, J.; Sander, F.G. Finite element simulation of in vivo tooth mobility in comparison with experimental results. J. Mech. Med. Biol. 2003, 3, 79-94. [CrossRef]

8. Jeon, P.D.; Turley, P.K.; Ting, K. Three-dimensional finite element analysis of stress in the periodontal ligament of the maxillary first molar with simulated bone loss. Am. J. Orthod. Dentofacial. Orthop. 2001, 119, 498-504. [CrossRef] [PubMed]

9. Kawarizadeh, A.; Bourauel, C.; Zhang, D.; Götz, W.; Jäger, A. Correlation of stress and strain profiles and the distribution of osteoclastic cells induced by orthodontic loading in rat. Eur. J. Oral. Sci. 2004, 112, 140-147. [CrossRef] [PubMed]

10. Poppe, M.; Bourauel, C.; Jäger, A. Determination of the elasticity parameters of the human periodontal ligament and the location of the center of resistance of single-rooted teeth a study of autopsy specimens and their conversion into finite element models. $J$. Orofac. Orthop Fortschr. Kieferorthopädie 2002, 63, 358-370. [CrossRef] [PubMed]

11. Penedo, N.D.; Elias, C.N.; Pacheco, M.C.T.; Gouvêa, J.P.D. 3D simulation of orthodontic tooth movement. Dent. Press. J. Orthod. 2010, 15, 98-108. [CrossRef]

12. Grieve, W.G.; Johnson, G.K.; Moore, R.N.; Reinhardt, R.A.; DuBois, L.M. Prostaglandin E (PGE) and interleukin-1 beta (IL-1 beta) levels in gingival crevicular fluid during human orthodontic tooth movement. Am. J. Orthod. Dentofac. Orthop. 1994, 105, 369-374. [CrossRef]

13. Sandy, J.R.; Farndale, R.W.; Meikle, M.C. Recent advances in understanding mechanically induced bone remodeling and their relevance to orthodontic theory and practice. Am. J. Orthod. Dentofac. Orthop. 1993, 103, 212-222. [CrossRef]

14. Singh, D.K.; Kumar, G. Comparison of the subgingival temperature of smokers and nonsmokers in healthy and diseased sites of gingiva in association with sublingual body temperature. J. Fam. Med. Prim. Care 2019, 8, 3166-3172. [CrossRef] [PubMed] 
15. Gunupati, S.; Sappiti, H.; Nagarakanti, S.; Reddy, B.R.; Chava, V.K. Validating gingival surface temperature as an alternative tool in the diagnosis of periodontal disease activity: An observational clinical trial. J. Dent. Res. Dent. Clin. Dent. Prospect. 2019, 13, 123-127. [CrossRef] [PubMed]

16. Lee, B. Relationship between tooth movement rate and estimated pressure applied. J. Dent. Res. 1965, 44, 1053. [CrossRef] [PubMed]

17. Storey, E.S. Force in orthodontics and its relation to tooth movement. Aust. J. Dent. 1952, 56, 11-18.

18. Sambataro, S.; Bocchieri, S.; Bafumi, L.; Fiorillo, L.; Cervino, G.; Cicciù, M. Elastics Selector Gauge as Orthodontics Device Applied to Inter-Maxillary Traction during Malocclusion Correction. J. Funct. Morphol. Kinesiol. 2019, 4, 63. [CrossRef] [PubMed]

19. Gioka, C.; Zinelis, S.; Eliades, T.; Eliades, G. Orthodontic latex elastics: A force relaxation study. Angle Orthod. 2006, 76, 475-479. [PubMed]

20. Kamisetty, S.K.; Nimagadda, C.; Begam, M.P.; Nalamotu, R.; Srivastav, T.; Gs, S. Elasticity in Elastics-An in-vitro study. J. Int. Oral Health 2014, 6, 96-105. [PubMed] 